\title{
Further Development of an Environmental HVTEM for Reaction Science by a New Non-exposure Transfer Holder
}

\author{
Nobuo Tanaka ${ }^{1}$, Shigeo Arai ${ }^{1}$ and Shigemasa Ohta ${ }^{2}$ \\ ${ }^{1}$ Institute of Materials and Systems for Sustainability, Nagoya University, Nagoya, Japan \\ 22-EM Business Unit, JEOL Co Ltd, Akishima, Japan
}

Environmental transmission electron microscopy(E-TEM) is one of the effective research methods for nano-materials in practical use, related to catalysts and battery electrodes[1]. The key-points for the electron microscopic observation are (1)gas-atmospheres in actual usage, (2)easily heating, (3)thicker samples prepared from actual devices and circuits, and (4)non-exposure to air for decrease of oxidation and hydration. The problems of ordinary environmental TEM still remain after the development of MEMS chip holders. For example, the electrodes of lithium battery should be FIB-fabricated and transferred to (S)TEM instruments without exposure to air, and the actual samples are rather thicker, which do not give clear-cut images by using medium-voltage TEM. In the present study we have developed a new transfer system from a glove box to E-HV(S)TEM.

The E-HV(S)TEM used is a 1 MV instrument developed in Nagoya University in collaboration with JEOL since 2008, where various kinds of in-situ observation such as heating, cooling, mechanical operation, gas reaction, ice-embedding are available with the maximum TEM resolution of $0.1 \mathrm{~nm}$ and STEM resolution of $1 \mathrm{~nm}[2]$. In the present study we have developed a transfer holder for non-exposure transfer as shown in Fig. 1. The holder has a special closing sleeve for non-exposure of samples to air. Also a special vessel is manufactured in order to transfer from a FIB apparatus to the glove box filled in dry inert gases. Figure 2 shows low and high-resolution images of a rhodium/graphite sample transferred by the system. Figure 3 shows E-HVTEM images of tin particles heated in $523 \mathrm{~K}$ after transferring with exposure to air. The image with the exposure showed very thin oxide layers on the particle surface. Then, after heating/melting, oxidation process was in-situ observed with injection of oxygen of $5 \times 10^{-4} \mathrm{~Pa}[3]$.

References:

[1] Dehm, Howe \& Zweck eds. "In-situ Electron Microscopy" (Wiley, 2012).

[2] N. Tanaka et al., Microscopy, 62(2013), p.205.

[3] The present authors would like to acknowledge Prof. S. Mutoh for warm allowance for the present use of the HV(S)TEM and Messrs. Y. Yamamoto, K. Higuchi and T. Adachi for collaboration. The authors acknowledge Grant-In-Aid from MEXT, Japan (26246006). 


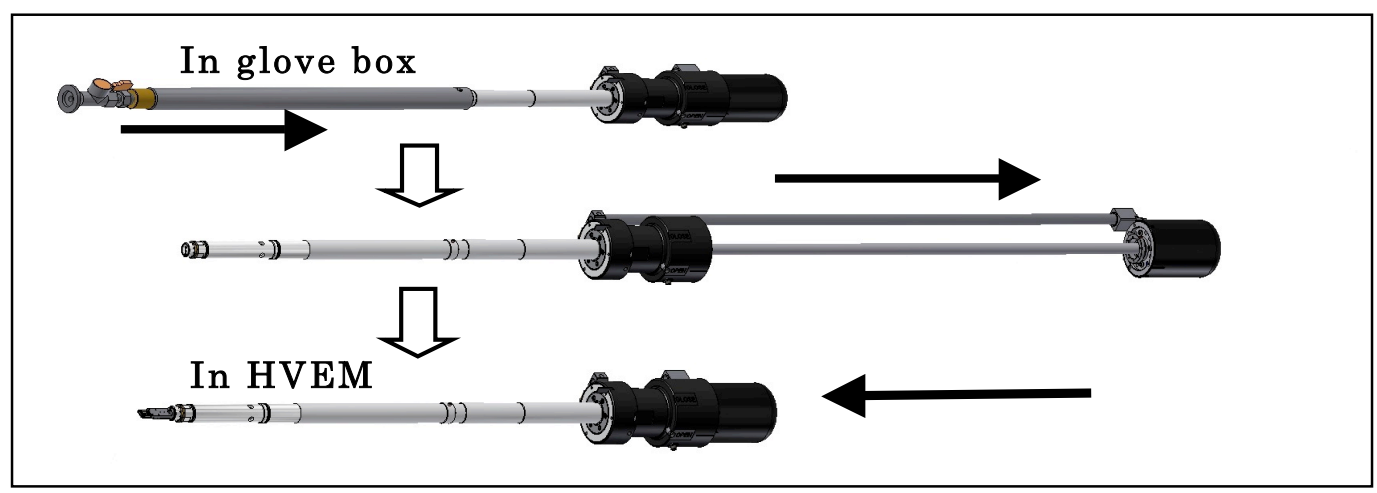

Figure 1. Photos of the present transfer holder with non-exposure to air.

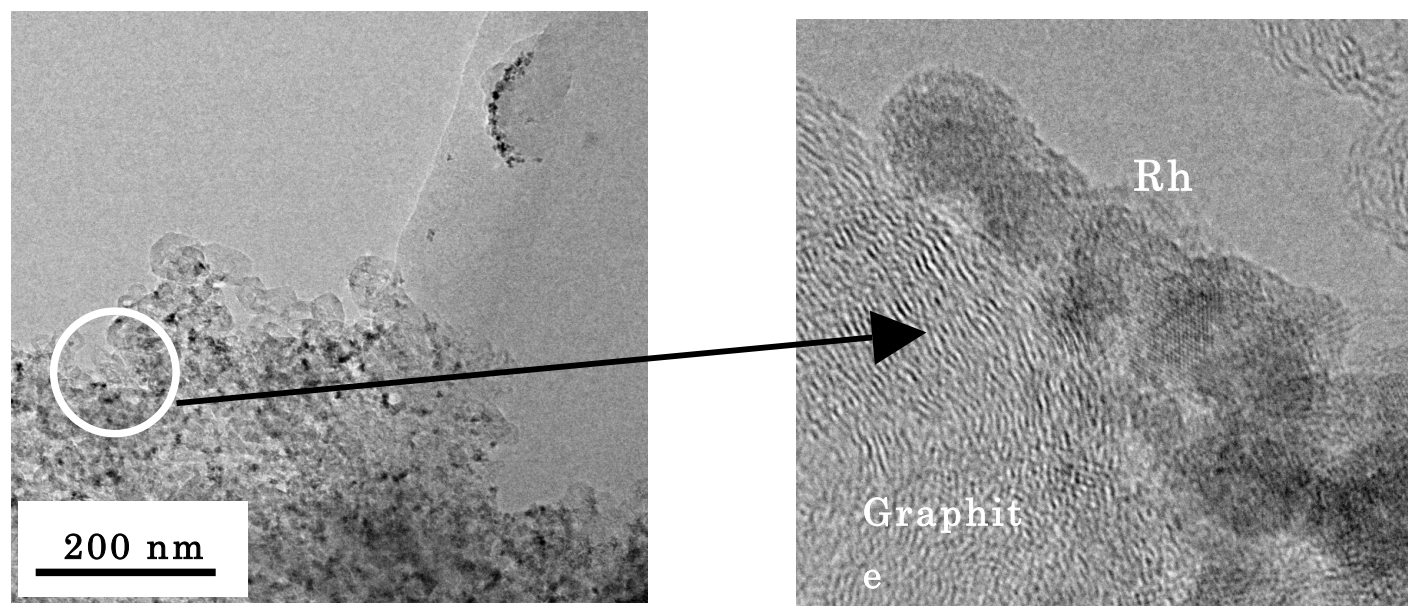

Figure 2. High resolution HVTEM images of an electrode sample of $\mathrm{Rh} /$ graphite, transferred by this new system at $1 \mathrm{MV}$ voltage.
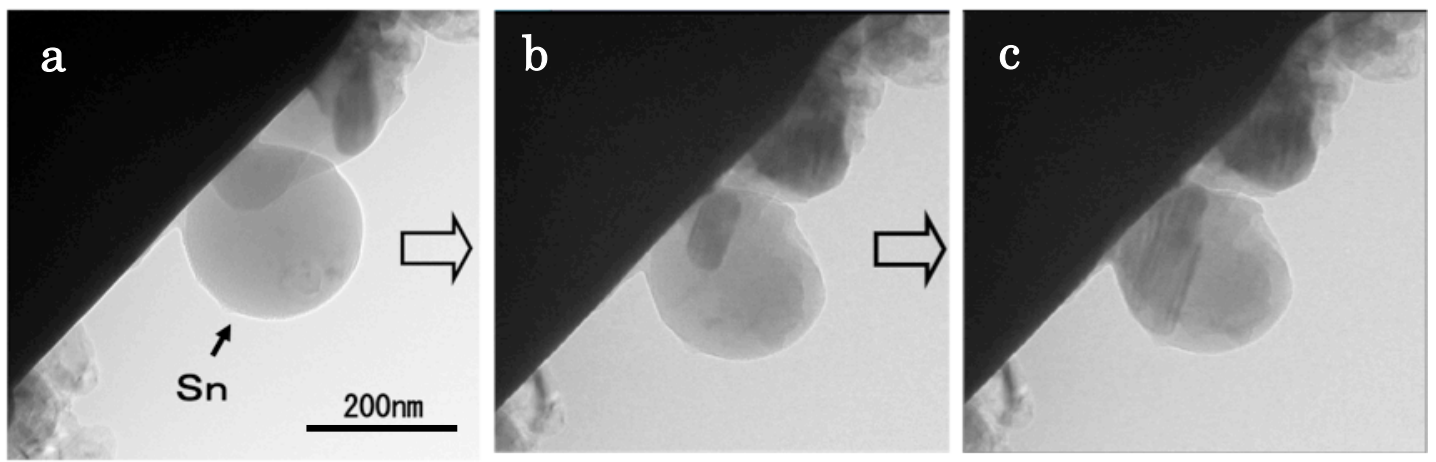

Figure 3. E-HVTEM images of oxidation of tin particles with injection of $5 \times 10^{-4} \mathrm{~Pa}$ of oxygen. 